\title{
W-Wort-Verdoppelung im Schweizerdeutschen
}

\author{
Natascha Frey (Zürich)
}

\begin{abstract}
In some Swiss German dialects, wh-questions can show the wh-word at the end of the sentence in addition to its 'normal' sentence initial position. This phenomenon called whdoubling raises some puzzling questions for linguistic theories, such as: what kind of processes are involved in wh-doubling (syntactic, phonological)? Does wh-doubling enrich the poor left periphery of Swiss German? Why do speakers use an additional wh-word that seems to be absolutely superfluous? I will argue that wh-doubling depends on the information structure of the question, more specifically on the function of the wh-word as a focus constituent. Wh-doubling is also used in a special type of rhetorical questions in Swiss High German where in addition to doubling wh-words undergo diminutive formation and reduplication. My paper pursues two main goals: (i) to give a detailed description of whdoubling constructions with regard to geographical distribution and question type (rhetorical, alternative, echo etc.); (ii) to present syntactic analyses of similar wh-doubling phenomena in other languages considering their application to Swiss German data.
\end{abstract}

\section{Das Phänomen und die Fragestellung}

Im Folgenden werden eine kurze Beschreibung der wh-doubling-Konstruktion im Urner Dialekt des Schweizerdeutschen gegeben sowie Fragen formuliert, denen in den nachfolgenden Abschnitten nachgegangen wird. Zum einen wird die w-Wort-Verdoppelung aus der Perspektive der Informationsstruktur analysiert. Zum anderen fragt es sich, welche syntaktische Prozesse dabei beteiligt sind.

\subsection{W-Wort-Verdoppelung im Urner Dialekt}

Bei der Beschreibung der w-Wort-Verdoppelung (wh-doubling) im Schweizerdeutschen nehme ich als Beispiel den Urner Dialekt (UD), in dem diese Satzkonstruktion zum ersten Mal beobachtet und beschrieben und anhand eines Datenkorpus ausführlich analysiert wurde (cf. Frey 2001). Das Datenkorpus besteht aus vier Audioaufnahmen von Spontangesprächen (je ca. 1 Stunde), die im Kanton Uri gemacht wurden. An den Gesprächen waren jeweils vier bis fünf Personen beteiligt. ${ }^{1}$

\footnotetext{
${ }^{1}$ Alle im Folgenden angeführten Beispiele stammen aus diesem Korpus, wenn nicht anders gekennzeichnet.
} 
Beim sogenannten wh-doubling handelt es sich um die Verdoppelung des w-Wortes in Ergänzungsfragen (w-Fragen). Im UD äussern die Dialektsprecher solche Sätze wie in (1):
(1) a. was machämer moorä?
b. was machämer moorä was?
c. wiä wotsch das machä wiä?
d. wenn hesch dü dyys Referat wenn?
e. was gfindet er nu alles ü̈̈sä was?
f. $\quad$ rä̈̈chsch dü was? = was rä̈̈chsch dü was? (wh-drop)

(1a) \& (1b) zeigen, dass das Fragewort am Ende des Satzes wiederholt werden kann. Im Kanton Uri ist die Form mit w-Verdoppelung bei einem Teil der Dialektsprecher die normale und unmarkierte Art und Weise, eine Frage zu stellen. Sie ist jedoch in dem Sinne nicht obligatorisch, als die Sprecher zunächst einmal je nach Gesprächssituation bzw. je nachdem wen sie als Gesprächspartner vor sich haben, frei entscheiden können, ob sie diese Form verwenden oder nicht. Wenn beispielsweise die Gesprächspartner einen anderen Dialekt sprechen, so wird die w-Verdoppelung von einem Teil der UD Sprechenden aus Anpassungsgründen vermieden. Wenn hingegen die Gesprächspartner dem Sprecher vertraut sind (Familienmitglieder, Freunde usw.), besteht eher die Tendenz die Frageform mit Verdoppelung zu verwenden.

Im UD sieht es so aus, dass ein Teil der Dialektsprecher die w-Verdoppelung aktiv und differenziert einsetzt. Dem anderen Teil ist diese Frageform nur passiv bekannt, d.h. sie hören sie von den anderen, verwenden sie aber selber nicht in gleicher Weise. Bei diesem anderen Teil wird das w-Wort in einem ganz speziellen (markierten) Kontext verdoppelt. Darauf werde ich im Abschnitt 4.1.1 zurückkommen.

Verdoppelt werden nicht nur pronominale w-Elemente wie wer oder was in (1), sondern auch adverbiale w-Wörter wie wiä und wenn wie (1c) \& (1d) zeigen. Ferner ist aus den Beispielen (1c) \& (1e) ersichtlich, dass die zweite Kopie des w-Wortes nicht in situ (d. h. nicht links von der rechten Verbalklammer) steht, sondern am rechten Rand des Satzes. In seltenen Fällen kann die erste Kopie am Anfang des Satzes ausfallen wie in (1f). Dieses wh-drop ist auch in anderen Schweizer Dialekten zu beobachten, beispielsweise dann, wenn im Diskurs eindeutig ist, wonach gefragt wird. ${ }^{2}$

Die w-Wort-Verdoppelung unterliegt folgenden Restriktionen:

1. verdoppelt werden ausschliesslich einsilbige $\mathrm{w}-\mathrm{W}$ örter wie was, wer, wiä, wenn, wo, die mehrsilbigen w-Phrasen wie z.B. welä, wasfirr, wiäso, warum, wohärä sind davon nicht betroffen, auch wenn das w-Wort getrennt werden kann, z.B. wo gömmer härä?

2. die Echo-Fragen vom Typ WO chasch dü mitgaa? mit starker Betonung auf dem wWort in der satzinitialen Position sind von der Verdoppelung ebenfalls ausgeschlossen.

\footnotetext{
2 Diese Aussage stützt sich auf meine persönliche Beobachtungen im Berndeutschen. Ferner wird das wh-drop in Arbeiten zum Spracherwerb z. B. in Zvi Penner (1992: 262-263) für das Berndeutsche sowie in Kyoko Yamakoshi (2002) für Schwedisch, Niederländisch und Deutsch und ASL (American Sign Language) behandelt.
} 
Echo-Fragen mit dem Fragewort in situ kommen im Datenkorpus nicht vor (z. B. Du gehst WO mit? ).

3. in Alternativfragen wie was macht de der da, Feeriä oder Schaffä? wird das w-Wort auch nicht verdoppelt.

Die zusätzliche Befragung von Urner Gewährspersonen zu diesen Restriktionen hat diese Ergebnisse bestätigt.

Eine weitere Beobachtung betrifft das prosodische Muster der Fragesätze mit wVerdoppelung. Es scheint so zu sein, dass in diesen Sätzen keine Pause eingelegt werden kann, bzw. wenn aus irgendwelchen Gründen eine Pause entsteht, wird der Satz neu angesetzt oder ohne Verdoppelung realisiert. Möglicherweise spielt die Distanz (Silben- und nicht Konstituentenanzahl) zwischen zwei w-Wörtern eine Rolle, d.h. je kürzer der Satz, desto besser kann verdoppelt werden. Ob die (Silben-)Länge des Satzes auf die Verdoppelung tatsächlich einen Einfluss hat, wurde von mir bis jetzt allerdings nicht systematisch untersucht. Auffallend ist auch die starke Betonung des finiten Verbs in Doubling-Sätzen begleitet vom fallenden Tonverlauf. Im UD ist dieses Betonungsmuster extrem ausgeprägt im Vergleich zu anderen Dialekten (cf. Clauss 1969: 33-34). Der Tonverlauf kann mit einer steigend-fallenden Intonation auf der letzten betonbaren Silbe des Satzes abgeschlossen werden. Diese Silbe ist insofern wichtig, als sie möglicherweise die Realisierung des zweiten w-Wortes am Ende des Satzes unterdrückt. ${ }^{3}$

\subsection{Fragestellung}

Zu der w-Verdoppelung im UD, wie oben beschrieben, stellen sich nun folgende Fragen:

1. Gibt es funktionale Regelmässigkeiten bei der w-Verdoppelung, und wodurch wird sie gesteuert?

2. In welcher strukturellen Position befindet sich das zweite w-Wort und wie ist es dorthin gelangt? (im Sinne der w-Bewegung in der generativen Grammatik)

Die Frage 1 wird im nächsten Abschnitt behandelt. Zu der Frage 2 werden in diesem Artikel nur ansatzweise Überlegungen im Abschnitt 5 angestellt.

\section{Pragmatische Perspektive}

\subsection{D-linking als Auslöser der w-Wort-Verdoppelung}

Um die erste in 1.2 gestellte Frage zu beantworten, versuche ich zuerst zu zeigen, wie nun die in 1.1 aufgeführten Restriktionen mit einer pragmatischen Theorie der Fokus-Struktur erklärt werden können.

Da die mehrsilbigen w-Phrasen von der Verdoppelung ausgeschlossen sind, wenden wir uns zunächst den einsilbigen zu. Ich gehe davon aus, dass die Verdoppelung bei den einsilbigen

\footnotetext{
${ }^{3} \mathrm{Zu}$ diesem Intonationsmuster sagte eine Gewährsperson: wenn ich den Satz so (steigend-fallend) ausspreche, muss ich dass zweite w-Wort nicht wiederholen.
} 
w-Wörtern im UD eine Default-Option darstellt, also ein unmarkierter Fall ist. Diese werden aber in bestimmten Diskurskontexten nicht verdoppelt (vgl. Restriktionen 2 und 3 in 1.1). Was sind das für Kontexte? Oder die Frage anders formuliert: Erfüllen die w-Wörter jeweils die gleiche Funktion im Satz? Das scheint nicht der Fall zu sein. Es wird allgemein angenommen, dass w-Wörter fokustragende Elemente sind. Dem ist aber nicht immer so. Nomi Erteschik-Shir (1997) zeigt in ihrer Arbeit über die Fokus-Struktur, dass die w-Wörter in bestimmten Gesprächssituationen als Topik interpretiert werden können. Fokus und Topik werden folgendermassen definiert: 4

(2) The topic is what a statement is about.

The topic is used to invoke "knowledge in the possession of an audience."

"The statement is assessed as putative information about its topic."

(Erteschik-Shir (1997: 9) mit Bezug auf Strawson (1964: 97))

(3) The Focus of a sentence $S=$ the (intension of a) constituent $\mathrm{c}$ of $\mathrm{S}$ which the speaker intends to direct the attention of his/her hearer(s) to, by uttering S.

(Erteschik-Shir 1997: 11)

Für meine Argumentation reicht es, das Topik auf die "alte" und den Fokus auf die "neue" Information im Diskurs zu reduzieren. Bei der Zuweisung der Fokus-Eigenschaft einer Konstituente gilt die Perspektive der sprechenden Person.

Auf die Fokuskonstituenten wird eine Betonungsregel angewandt, derzufolge die Fokuskonstituenten betont sein müssen:

(4) Assign stress to the focus constituents.

Diese Regel gilt zunächst einmal für das Englische (cf. Erteschik-Shir 1997: 124) und für das Dänische (cf. Erteschik-Shir/Strachov (2004: 303). Vermutlich gilt die Regel auch für andere Sprachen, in denen der Fokus mit musikalischer Betonung (pitch accent) markiert wird (Erteschik-Shir, p.c.). Der UD gehört zu diesen Sprachen, wie die Grammatik von Walter Clauss (1967: 30-37) es bezeugt. Ich nehme also an, dass diese Betonungsregel auch auf den UD angewandt werden kann.

In w-Fragen können die w-Wörter in den sogenannten restriktiven Kontexten als Topik fungieren (cf. Erteschik-Shir 1997: 105-107). Solche Kontexte liegen vor, wenn die mögliche Antwort aus dem Gespräch erschliessbar oder bekannt ist, z.B. bei Alternativfragen, rhetorischen Fragen und bei Negativfragen. Nehmen wir als Beispiel die Alternativfrage in (5):

(5) A: was macht de der da, Feeriä oder Schaffä?

B: der isch z Bsuäch da.

(5) zeigt, dass der Set der möglichen Antworten auf die Frage, die mit was eingeleitet ist, durch den Kontext, hier durch Feeriä oder Schaffä, restringiert wird. In diesem Fall ist das w-

\footnotetext{
${ }^{4}$ Es kann hier auf die Theorie der Fokus-Struktur nicht genauer eingegangen werden. Deshalb beschränke ich mich auf die für w-Fragen relevanten Aspekte.
} 
Wort diskursgebunden (d-linked). Die gleiche Überlegung würde auch für den Satz ohne den Set Feeriä oder Schaffä gelten, wenn diese Information vorher im Gespräch erwähnt wurde. ${ }^{5}$

Da in Alternativfragen im UD keine w-Verdoppelung zu beobachten ist, drängt sich dafür folgende Erklärungshypothese auf: nur diejenigen w-Wörter werden verdoppelt, die als Fokus fungieren, nicht aber solche mit Topik-Eigenschaft.

Die Analyse der Fragesatzsequenzen im UD nach diesem Kriterium (Fokus- oder Topik-Interpretation) ergibt, dass die Verdoppelung gerade in solchen restriktiven Kontexten (w-Wort als Topik) ausgeschlossen ist, d. h. verdoppelt wird nur ein w-Wort in Fokusfunktion. Das würde die Restriktion 3 erklären (s. S. 137).

Laut der Betonungsregel muss die Fokuskonstituente betont sein. In den Sätzen, in denen die Fokuskonstituente aus einer Silbe besteht (was, wiä usw.) ist es nicht möglich, sie zu betonen, weil das w-Wort sich prosodisch an das finite Verb, das die stärkste Betonung im Satz trägt, unterordnet. Das könnte der Grund für die Verdoppelung sein: Die Fokuskonstituente wird durch die Verdoppelung um eine Silbe erweitert, und diese Silbe, das w-Wort am Ende des Satzes, hat phonetisch gesehen mehr Gehalt, als die Kopie am linken Rand. Es wird nicht durch die Klitisierung dermassen reduziert, wie dasjenige am linken Rand. ${ }^{6}$

Was ist nun mit mehrsilbigen w-Phrasen und mit den Echo-Fragen? Die mehrsilbigen wPhrasen werden nicht verdoppelt, weil sie dank ihrer Mehrsilbigkeit eine eigene Betonung tragen. In Echo-Fragen wird das w-Wort ohnehin stark betont und braucht deshalb nicht verdoppelt zu werden. So scheint die Verdoppelung von einsilbigen w-Elementen in FokusFunktion eine Art Ersatz für Betonung zu sein, um das w-Wort als Fokus zu markieren. Das wäre eine Erklärung für die Restriktionen 1 und 2 (s. S. 136f.).

Für die Verdoppelung des w-Wortes im UD sind also folgende Faktoren entscheidend: Silbenzahl der Fokus-Konstituente und Fokus-Topik-Eigenschaft des w-Wortes. Nur die einsilbigen w-Wörter, die als Fokus fungieren, können verdoppelt werden. Diese zwei Faktoren können im Prinzip auf die Anwendung der Betonungsregel für die Fokuskonstituenten reduziert werden.

\section{Geographische Verteilung der w-Wort-Verdoppelung}

In 1.1 habe ich erwähnt, dass die W-Wort-Verdoppelung ein Mittel ist, das im Dialekt zur Verfügung steht und von Individuen selektiv eingesetzt werden kann. In 2.1 habe ich dafür argumentiert, dass die w-Verdoppelung dazu dient, das W-Wort als Fokus zu markieren. Dazu kann sich einem die Frage aufdrängen: wieso machen das die anderen Dialekte nicht? Zuerst muss aber überprüft werden, ob die Präsupposition in der Frage überhaupt stimmt. Es stellt sich also die Frage, ob überall in der Deutschschweiz verdoppelt werden kann. Und die

\footnotetext{
${ }^{5}$ Auf die rhetorischen Fragen und auf die Negativfragen gehe ich hier nicht ein, weil sie im Urner Korpus nicht enthalten sind. Das wird aber das Thema weiterer Untersuchungen sein.

${ }^{6}$ Es ist aber nicht ganz klar, ob die linke Kopie des w-Wortes reduziert wird oder ob eher die rechte Kopie gedehnt wird durch die sogenannte präpausale Dehnung (final lengthening).
} 
anschliessende Frage: wo wird gegebenenfalls die Verdoppelung der "normalen" Frageform bevorzugt?

Da das wh-doubling, wie gesagt, als Option individuell gebraucht werden kann (da müsste man auf die "richtigen" Informanten treffen) und diskursgesteuert wird (da muss man hoffen, dass die Informanten sich in die vorgegebene Gesprächssituation einleben können), nehme ich an, dass es an vielen Orten eine gewisse Akzeptanz findet und dass diese Akzeptanz eher niedrige Werte aufweisen wird.

\subsection{Abfrage per Fragebogen}

Im Folgenden stütze ich mich auf die Daten, die im Nationalfondsprojekt 'Dialektsyntax des Schweizerdeutschen' an der Universität Zürich erhoben wurden. ${ }^{7}$ Das Phänomen wurde im 4. Fragebogen an insgesamt 383 Orten in der Deutschschweiz abgefragt. Die relevante Frage wird mit einer Kontextangabe eingeleitet, die das w-Wort in seiner Fokus-Funktion wie im UD fungieren lassen soll:

III.26 Ihr Nachbar Urs ist vor Jahren nach Amerika ausgewandert. Béatrice hat ihn kürzlich besucht. Sie fragen sie:

\&elche der folgenden Sätze können Sie in Ihrem Dialekt sagen ("ja"), welche sind nicht möglich ("nein")?

ja nein

1) $\square$ Was macht de Urs jetzt?

2) $\square$ Was macht de Urs jetzt was?

3) anders:

Welche 'Ja'-Variante (1-3) ist für Sie die natürlichste?

Nr.

Abb. 1: Ausschnitt aus dem 4. Fragebogen des Projekts 'Dialektsyntax des Schweizerdeutschen', Frage Nr. 23

Die Gewährspersonen sind aufgefordert, ein Urteil über die Grammatikalität der w-Fragen mit und ohne w-Verdoppelung abzugeben. Dabei muss die Entscheidung nicht ausschliessend sein, d. h. es können beide Varianten (1 und 2) z. B. mit 'ja' angekreuzt werden. Wenn die Frage unter 2 im 'ja'-Feld angekreuzt wird, so ergibt sich daraus ein Bild der Akzeptanz der Verdoppelung. Unter 3 steht eine Option zur Verfügung für den Fall, dass keine der beiden Fragen in 1 und 2 in den vorgegebenen Kontext passen sollte. Und am Schluss wird nach der natürlichsten Variante gefragt. Wird zu dieser Frage 2 angegeben, wird die Variante 2 bei der Auswertung als präferiert verstanden.

\footnotetext{
${ }^{7}$ Näheres zum Projekt und zu den Methoden s. in Claudia Bucheli/Elvira Glaser (2002) und Claudia Bucheli Berger (in diesem Band).
} 


\subsection{Akzeptanz}

Die Werte für die Akzeptanz ergeben sich durch solche Antworten, in denen unter 2 'ja' angekreuzt wurde, unabhängig davon, welche Variante als die natürlichste angegeben wurde. An 78 Orten wurde die w-Verdoppelung als möglich bezeichnet. 17 Orte weisen mehr als eine Nennung auf. Die höchsten Werte (über 20\%) bei der Akzeptanz ohne Einzelnennungen wurden an folgenden Orten erzielt: ${ }^{8}$

\begin{tabular}{lccc} 
Dialekt von & positive Antworten & alle Antworten & Verhältnis \\
\hline NW Buochs & 4 & 7 & $57 \%$ \\
VS Betten & 2 & 4 & $50 \%$ \\
OW Melchtal & 2 & 5 & $40 \%$ \\
SZ Oberiberg & 2 & 5 & $40 \%$ \\
OW Alpnach & 3 & 9 & $33 \%$ \\
UR Altdorf & 3 & 9 & $33 \%$ \\
OW Sarnen & 2 & 7 & $29 \%$ \\
LU Ruswil & 2 & 7 & $29 \%$ \\
LU Eschenbach & 2 & 7 & $29 \%$ \\
NW Wolfenschiessen & 3 & 12 & $25 \%$ \\
UR Isenthal & 3 & 12 & $25 \%$ \\
LU Entlebuch & 2 & 8 & $25 \%$ \\
LU Sempach & 2 & 9 & $22 \%$ \\
UR Unterschächen & 2 & 9 & $22 \%$ \\
ZG Oberägeri & 2 & 9 & $22 \%$ \\
\hline
\end{tabular}

Tab. 1: Akzeptanz der w-Verdoppelung (ohne Einzelnennungen)

Die Prozentzahlen dienen der ungefähren Orientierung, da die Gesamtzahl der Gewährspersonen pro Ort z. T. sehr tief ist.

Auf der nachfolgenden Karte sind alle Orte angegeben, wo die w-Verdoppelung akzeptiert wurde, d.h. auch die Orte mit Einzelnennungen. Sie sind mit kleinen Symbolen gekennzeichnet.

Die Tabelle und das Bild zeigen, dass die Orte mit der höchsten Akzeptanz sich in der Innerschweiz konzentrieren, und zwar in den Kantonen Nidwalden, Obwalden, Schwyz, Uri und Luzern. Der Kanton Uri ist durch zwei Orte vertreten, deren Werte im mittleren Bereich liegen. Erstaunlicherweise zeigt Betten im Wallis die zweithöchste Akzeptanz. Allerdings ist hier die niedrige Gesamtzahl zu berücksichtigen. Die niedrigeren Akzeptanzwerte (22-33\%) fallen ebenfalls auf die Zentralschweiz (s. Tab 1). Es sieht so aus, dass in den Dialekten dieser Region die w-Verdoppelung als eine ernstzunehmende Option zur Verfügung steht. In der übrigen Schweiz ist die Akzeptanz überall etwa gleich niedrig, im Berner Oberland scheint sie sogar zu fehlen.

\footnotetext{
${ }^{8}$ Die quantitativen Angaben beziehen sich auf den momentanen Stand der Auswertung (August 2004) der ca. 2500 Fragebögen. Diese Daten dienen als Grundlage für die in 3.2 und 3.3 abgebildeten Karten.
} 


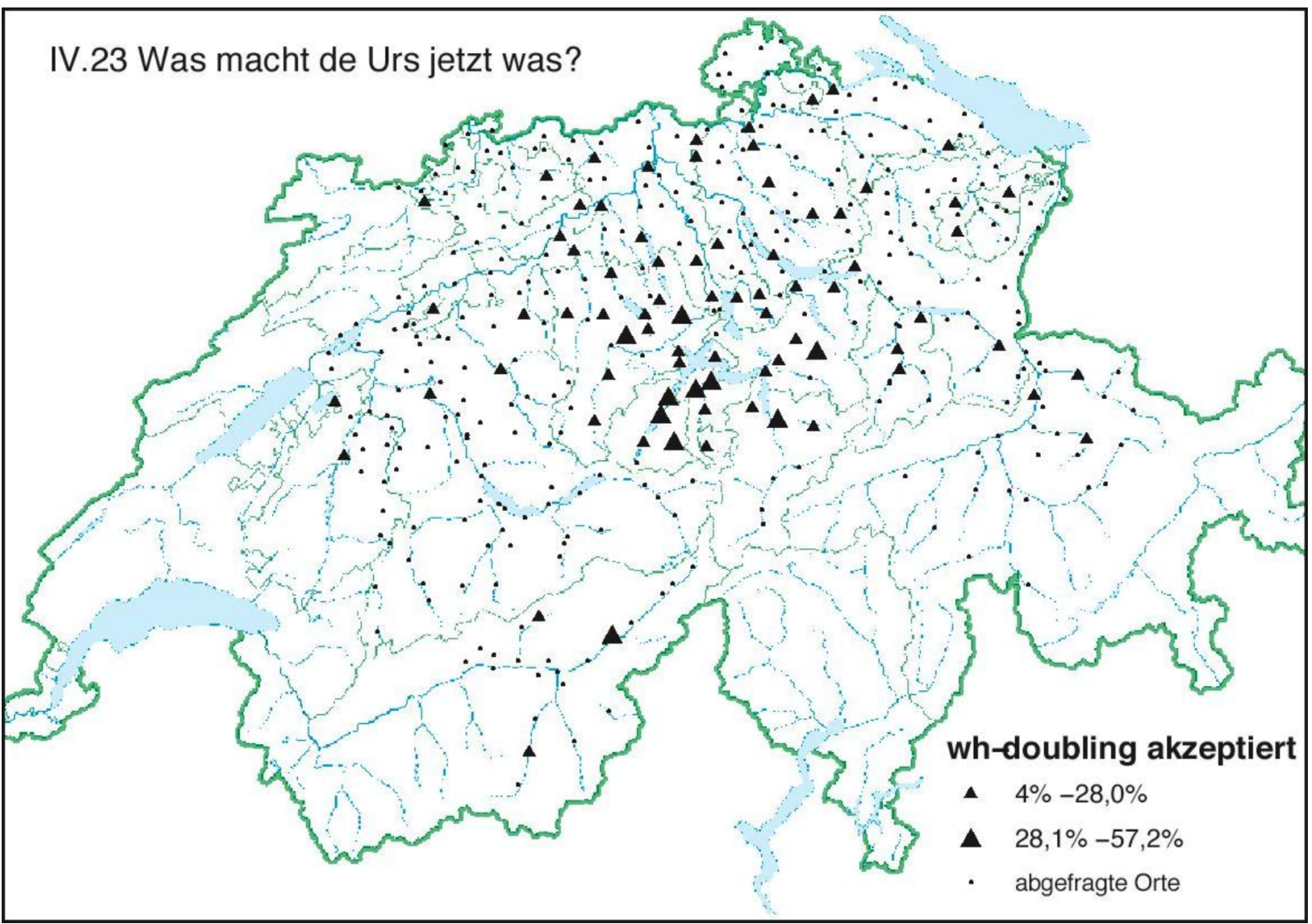

Karte 1: Orte mit Akzeptanz der w-Verdoppelung

\subsection{Präferenz}

Als Präferenz werden solche Antworten gewertet, in denen als natürlichste Variante der Satz mit w-Verdoppelung angegeben wurde. Hier liegen die höchsten Werte in den Kantonen Nidwalden und Obwalden. Ohne Einzelnennungen sieht die Datenlage folgendermassen aus:

\begin{tabular}{lccc} 
Dialekt von & positive Antworten & alle Antworten & Verhältnis \\
\hline NW Buochs & 4 & 7 & $57 \%$ \\
NW Stans & 2 & 5 & $40 \%$ \\
OW Alpnach & 3 & 9 & $33 \%$ \\
OW Sarnen & 2 & 7 & $29 \%$ \\
NW Wolfenschiessen & 3 & 12 & $25 \%$ \\
LU Sempach & 2 & 9 & $22 \%$ \\
UR Isenthal & 2 & 12 & $17 \%$ \\
\hline
\end{tabular}

Tab. 2: Präferenz der w-Wort-Verdoppelung ohne Einzelnennungen

Die höchsten Werte zeigen wiederum die Orte in der Zentralschweiz. Im Kanton Uri hat ausser in Isenthal noch eine Gewährsperson in Unterschächen die w-Verdoppelung präferiert. 
Auf der folgenden Karte sind wiederum auch die Orte mit Einzelnennungen berücksichtigt. An den Orten, wo die w-Verdoppelung präferiert wird, haben von 192 Gewährspersonen 34 diese als natürlichste Variante bezeichnet. Das macht $17,7 \%$ aus.

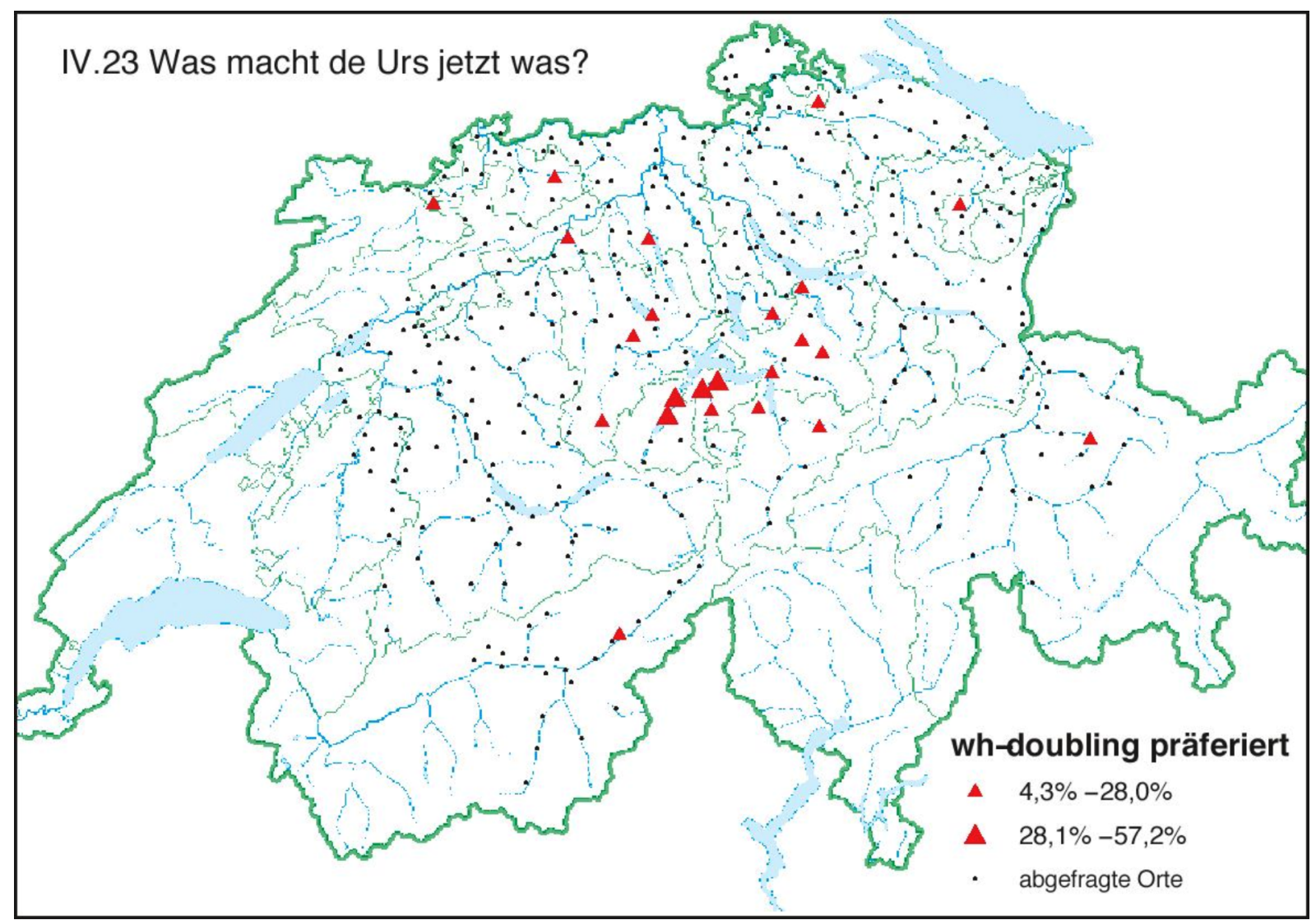

Karte 2: Orte mit Präferenz der w-Verdoppelung

Wenn man die Daten der Präferenz und der Akzeptanz ohne Einzelnennungen miteinander vergleicht, so wird an sechs Orten, wo die Verdoppelung akzeptiert wird, diese im gleichen Masse auch präferiert (Buochs, Stans, Alpnach, Sarnen, Wolfenschiessen, Sempach).

Im grossen und ganzen hat die Abfrage des Projekts 'Dialektsyntax des Schweizerdeutschen' deutlich gezeigt, dass das wh-doubling praktisch überall in der Deutschschweiz mit Ausnahme von Berner Oberland wenn auch im niedrigen Masse akzeptiert wird. Dieses Bild entspricht sehr gut den Merkmalen der w-Verdoppelung wie Optionalität und Diskursgebundenheit, die der obigen Annahme zufolge zwar ein breites Areal, dafür aber niedrige Werte ergeben.

\section{Variation der w-Wort-Verdoppelung im Schweizerdeutschen}

In diesem Abschnitt wird anhand anderer Beispiele gezeigt, wie die w-Verdoppelung im Schweizer(hoch)deutschen auf eine andere Art und Weise realisiert werden kann. Es handelt sich um rhetorische Kontexte. Unter rhetorischen Fragen verstehe ich im weiteren Sinne solche Fragen, auf die keine Antwort von der fragenden Person erwartet wird. 


\subsection{Rhetorische Fragen}

\subsubsection{Emotionale Einstellung zum Thema}

Im Abschnitt 1 wurde erwähnt, dass die die w-Verdoppelung im UD nur bei einem Teil der Dialektsprecher in Gebrauch ist. Dem anderen Teil ist diese Satzkonstruktion ebenfalls bekannt, nur in einem anderen Kontext. Stellen wir uns vor, Person A kocht gerade Milch und sie wird einen Moment durch etwas abgelenkt. Person B beobachtet, wie die Milch überläuft. In dieser Situation kann sich Folgendes abspielen:

(6) B: was machsch dü was?!

A: oh!

In (6) stellt B eine Frage, die eine starke emotionale Färbung annimmt (Erstaunen oder Vorwurf). Diese Äusserung hat eher einen rhetorisch-exklamativen Charakter, weil es ja offensichtlich ist, was die andere Person macht. Somit liegt hier keine echte Frage vor wie z. B. in (1). Diese Art von wh-doubling in gefühlsbetonten Äusserungen (keine kanonischen Fragen) ist auch in einigen norditalienischen Dialekten verbreitet, wobei die w-Verdoppelung nicht obligatorisch ist, wie das Beispliel aus Nicola Munaro (2003) zeigt:

(7) $\quad \operatorname{cossa}$ a-tu fat (che)? (Bellunese - North. Veneto)

What have you done what

'what have you done what?!'

\subsubsection{Vermutung über das Erfragte}

Beim Beispiel (8) muss man sich eine Situation vorstellen, in der die fragende Person die Antwort nicht weiss, wie in (7), sondern vermutet.

(8) was macht er was/waseliwas?

Wie in (7) wird auch hier keine echte Frage gestellt, vielmehr wird hier die Vermutung bis hin zu Sicherheit über die mögliche Antwort zum Ausdruck gebracht. Sätze wie in (4.1.2) werden von Sprechern des Berndeutschen und anderer Dialekte akzeptiert. Sehr häufig wird in diesem Zusammenhang das Sprechen mit kleinen Kindern erwähnt, wo solche Fragepronomina wie waseliwas besonders häufig gebraucht werden.

\subsubsection{Waseliwas}

Obwohl von den Sprechern selbst nicht selten als Kindersprache oder in die Kabarettperformance abgetan, werden die reduplizierten w-Wörter wie waseliwas dennoch auch in Kontexten gebraucht, die man nicht unbedingt in Verbindung mit Babytalk bringen würde..$^{9}$ Nach waseliwas-Muster (9a) werden auch andere einsilbigen w-Wörter modifiziert. Man findet beispielsweise wereliwer (9b), woseliwo/wodeliwo $(9 \mathrm{c})$, wenneliwenn und wiedeliwie:

\footnotetext{
${ }^{9}$ Verweise auf Kindersprache im Zusammenhang mit waseliwas und ähnlichen Bildingen finden sich in auch in Mundartgrammatiken und Wörterbüchern (cf. Suter 1995, von Greyerz 1976, Seiler 1879 und Muster 2001).
} 
(9) a. Und was passiert nach unseren schauerlichen mittelalterlichen Vorstellungen denn dort unten in der Hölle, waseliwas? Eben. Anbraten! [...] (<http: //www.salz-pfeffer.ch/suparch/01-03/story.html> 14.02.2003)

b. Unsere Politiker dagegen sind internetmässig gesehen, immer noch Hinterwäldler. Wer, wereliwer ist aber den von ihm "classe politique" geschimpften Kolleginnen und Kollegen "oben in Bern" voraus: Der Blocher, Christoph. [...] (<http://www.pctip.ch/topthema/tt/14918.asp> 14.02.2003)

c. Zum Beispiel die Sparmassnahmen: wird jetzt gespart oder nicht? Selbstverständlich wird gespart, wo sind wir denn? Und wo wird denn gespart, woseliwo? $\mathrm{Na}$ in der Schule eben. [...] (<http: //www.muensingen.ch/elternrat/kolumne/beitraege.html> 14.02.2003)

Bemerkenswert an diesen Beispielen (ausser 9d) ist einerseits der Gebrauch der dialektalen Fragewörter in der Schriftsprache, also im Hochdeutschen. Andererseits handelt es sich in (9) um rhetorische Fragen, genauer gesagt um Fragen, die keine neue Information von der gefragten Person verlangen und in denen das Fragewort auf jemand oder etwas referiert, was der angesprochenen Person nicht unbedingt bekannt sein muss aber naheliegend sein kann. Im Gegensatz zu kanonischen rhetorischen w-Fragen tritt bei denjenigen in (9) nie die Partikel schon auf, und es finden sich keine Fragen mit waseliwas-Muster, die als negative Existenzaussage, z. B. Wer zahlt schon gern Steuern? (Niemand), oder als positive Allaussage z. B. Wann ist er schon nicht am essen? (Er ist doch immer am essen) interpretiert werden können. Vermutlich sind solche Sätze mit waseliwas gar nicht möglich, weil das Fragewort sehr konkret für ein bestimmtes Thema steht.

Prosodisch unterscheiden sich die Sätze in (9) von denjenigen in (1) im Abschnitt 1 dadurch, dass vor dem w-Wort am rechten Rand des Satzes (waseliwas) eine Pause eingelegt werden kann. Dieses prosodische Muster ist typisch für die tag-Sequenzen, wie z. B. Er hat doch sein Haus verkauft, oder nicht?

Aus der morphologischen Perspektive weist waseliwas ein interessantes Wortbildungsmuster auf: was-eli-was $=$ Fragepronomen-Diminutivsuffix-Fragepronomen. Wenn man davon ausgeht, dass es sich beim w-Wort um ein funktionales Element handelt, fallen dazu zwei Dinge auf. Erstens werden Diminutiva normalerweise nur von lexikalischen Elementen gebildet, mit Fragepronomina ist diese Modifikation nicht üblich und wahrscheinlich auch nicht möglich, abgesehen von Beispielen in 4.1.3. Das Fragewort wo hat zwei Formen woseli und wodeli. In diesen beiden Ableitungen sowie in wiedeliwie dient der Bindekonsonant $-d-/-$ $s$ - als Hiatus-Tilger zwischen dem Stamm und dem Diminutivfuffix. Das hiatustilgende $-s$ scheintaber unmotiviert zu sein. Als Erklärung wäre hier anzunehmen, dass es auf der Ebene der Silbenstruktur zu einer Übertragung per Analogie gekommen ist: wa-se-li >wo-se-li. Es gibt in schweizerdeutschen Dialekten andere Beispiele für Diminutiva von nicht-lexikalischen Elementen, wie z. B. sooli oder soodeli, sefili zu sefil 'so viel' (cf. Odermatt 1903: 88), duuli 
$d u$ (cf. Fischer 1960: 465), nü̈̈teli auch nominal nes goldigs Nü̈̈teli im Sinne von 'eine Kleinigkeit', gebraucht (cf. Fischer 1960: 456). ${ }^{10}$

Zweitens ist die Reduplikation des Fragepronomens ebenfalls nicht sehr verbreitet in den Sprachen der Welt. Auch in Sprachen, in denen die Reduplikation im allgemeinen sehr produktiv ist, z. B. in australischen Sprachen, wird sie auf Pronomina nicht angewandt. Eine Ausnahme stellt in dieser Hinsicht Yukulta dar, eine Sprache, die in Nordaustralien gesprochen wird. In Yukulta bezeichnet die Reduplikation der Pronomina eine Art Pluralität (cf. Fabricius 1998: 54-55): ${ }^{11}$

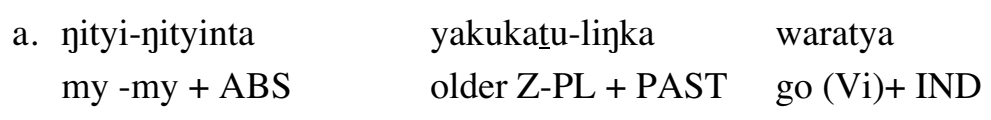

'All my sisters have gone'

b. நaka-yakaya-lkari kuritya tatint jawu

who?-who?ERG-they+PRES see+IND that+ABS dog

'Who are those people looking at that dog?'

(Yukulta, S. Keen 1983: 208)

Eine andere Sprache, die die Reduplikation der w-Wörter zulässt, ist das Koreanische. Im Koreanischen drückt die Reduplikation einerseits Pluralität aus, ohne dass das Fragepronomen als indefinit interpretiert werden kann (11a) ${ }^{12}$ Andererseits verleiht die Reduplikation dem w-Wort keine Frageinterpretation und das w-Wort muss singularisch interpretiert werden (11b) (cf. Chung 1999: 282-283):
a. John-i mwe-mwe kacyeo-ess-ni?
J.-Nom what-what bring-Pst-QE
'What (Pl) did John bring?'
\# 'Did John bring any / some things?'
b. yeysnal-ey sokumcangswu-ka eti-eti-lul ka-ess-nuntey, $\ldots$ long;time; ago salt;seller-Nom where-where-Acc go-Pst-When

'A long time ago, a salt seller went to a place, ...'

\# 'A long time ago, a salt seller went to some (several) places, ...'

Ausserdem erlaubt das Koreanische die sogenannten wh-triplets und wh-quadruplets. Fragesätze mit solchen Elementen haben einen Quiz-Fragen-Charakter, und die Zahl der wEinheiten stimmt mit der Zahl der vorausgesetzten Antworten überein (cf. Chung 1999: 284). ${ }^{13}$

Im Schweizerdeutschen scheint die Reduplikation mit der Pluralität nichts zu tun zu haben, sehr wohl aber mit der Vermutung oder mit dem Wissen über die mögliche Antwort. Die

\footnotetext{
10 Diminutivbildungen von ähnlichen Frageprononima und Adverbien (was, wer, niemand, so) finden sich auch in der russischen Umgangssprache.

${ }^{11}$ Zusätzlich zu Yukulta weisen folgende Sprachen reduplizierte w-Wörter auf: Ainu, Khmer, Mongolisch und Tagalog (cf. Haspelmath 1997: 180 Anm. 10).

${ }^{12}$ Im Koreanischen sind die w-Elemente ambig: sie können je nach Kontext und Intonation als w-Wörter oder als Indefinitpronomina interpretiert werden.

${ }^{13}$ Zur w-Reduplikation im Koreanischen siehe auch Cedric Boeckx (2000).
} 
emotionale Einstellung der fragenden Person zum erfragten Sachverhalt oder zu den angesprochenen Personen kommt hinzu, ausgedrückt durch den Gebrauch des Diminutivs. Zur Diminutivbildung ist noch zu bemerken, dass dabei das Suffix -eli und nicht -li, was ebenfalls möglich wäre, gebraucht wird. Ferner, die Ableitungen wie waseliwas und wodeliwo haben keinen Umlaut. Das Suffix -eli in Kombination mit dem fehlenden Umlaut scheint zumindest im Baseldeutschen einen stärkeren Gefühlsgehalt zum Ausdruck zu bringen:

An und für sich besteht kein Bedeutungsunterschied zwischen den Ableitungen auf -li und -eli, sofern sie durch die Form des Grundwortes (siehe (345) bedingt sind. Treten aber beim gleichen Grundwort beide Ableitungen auf, so hat diejenige mit -eli einen stärker verkleinernden Charakter, ein Maiteli ist also kleiner als ein Maitli, ein Hèmeli kleiner als ein Hèmli Hemdchen.

-eli hat weiteste Verbreitung in der Sprache, die Erwachsene kleinen Kindern gegenüber brauchen; den so gebildeten Verkleinerungen haftet etwas Zärtlich-Läppisches an: Hèt s Kindeli jètz wider waarmi Fiesseli? Kùmm, mer dien em Hanseli d Zeeneli bùtze. Durch das Ausbleiben des Umlauts wird dieser eher unbaslerische Gefühlsgehalt noch verstärkt: Jee, s Schätzli hèt kalti Handeli! (Rudolf Suter 1976: 201) ${ }^{14}$

Im Falle der Fragewörter könnte man annehmen, dass die Diminutivbildungen vom Typ waseliwas nicht so sehr die Grösse des modifizierten Wortes, sondern eher eine (positive) emotionale Einstellung des Sprechers ausdrücken und zwar durch die Suffigierung von -eli an den umlautlosen Stamm. In (9) hat sich der Gebrauch der reduplizierten w-Wörter mehr oder weniger grammatikalisiert, da nicht jeder Satz eindeutig in Verbindung mit positivem Gefühlsgehalt gebracht werden kann, wenn man z. B. (9a) mit (9c) vergleicht.

Auf der Satzebene kann die Position des reduplizierten Fragewortes variieren. Während in (9a), (9c) die w-Elemente sich jeweils am rechten und linken Rand des Satzes befinden, folgen die w-Wörter in (9b) unmittelbar aufeinander am linken Rand. In (9d)beginnt der Satz im Gegensatz zu anderen Beispielen in (9) mit waseliwas.

Die folgenden Beispiele zeigen das reduplizierte Fragewort in situ und ohne Verdoppelung:

(12) a. Übrigens: Laut Auskunft der bündner Regierung wurden in Landquart (WEF 2004) angeblich gar keine "Schockgranaten" eingesetzt, sondern nur <<Irritationswurfkörper $>>$, siehe Interview woz.ch. Nur: Unter der Bezeichnung Irritationskörper bietet die Herstellerin Nico auf ihrer Homepage - Überraschung - waseliwas wohl an? Na? Richtig: Blend-

SChock-Wurfkörper - na so ein Zufall [...]

(<http: //www.ssi-media.com/pigbrother/Report2003Part2.htm> 15.07.2004)

b. Habe mir noch schnell die Mühe gemacht und in diese pdf Datei hineingeschaut.

Euer Handy funktioniert nur unter 95/98/ME/2000 und waseliwas NICHT XP!!! Tut mir leid für euch. mfg Gast A

(<http: //irda.help4pc.de/1/msg/283981/283981/> 15.07.2004)

Fragesätze mit dem Fragewort in situ sind in Schweizer Dialekten im Gegensatz zum Hochdeutschen meiner Einschätzung nach nicht üblich. In der Schriftsprache (12a) wird diese

\footnotetext{
14 Dieselbe Meinung vertreten Ludwig Fischer (1960: 461-462) für das Luzerndeutsche und Albert Weber (1948: 328-329) für das Zürichdeutsche.
} 
Möglichkeit offenbar genutzt, und zwar so, dass das stark betonte w-Wort, das für EchoFragen Normalfall ist, durch ein redupliziertes w-Wort ersetzt wird. Dieses Mittel ist insofern für die geschriebene Sprache gut geeignet, als man die Betonung in der Schrift nicht so gut (dass es für alle verständlich wäre) wiedergeben kann. In (12b) stammt der Text von einer Web-Seite aus Deutschland. Es ist aber anzunehmen, dass der Eintrag von einem Schweizer oder einer Schweizerin geschrieben wurde. Waseliwas ist hier wohl als eine Art Parenthese zu verstehen.

In rhetorischen Kontexten bringt die Verdoppelung einerseits die emotionale Einstellung zum Thema des Gesprächs zum Ausdruck und andererseits die Vermutung oder das Wissen der fragenden Person über die mögliche Antwort. Dabei scheint die Möglichkeit der negativen Existenzaussage und der positiven Allaussage, die als Hauptarten rhetorischer Fragen angesehen werden, ausgeschlossen zu sein.

\section{$5 \quad$ Syntax der w-Wort-Verdoppelung}

In diesem Abschnitt möchte ich auf die Frage nach der syntaktischen Position der w-Kopie am rechten Satzrand eingehen. Ich stelle zwei für die Analyse der w-Verdoppelung im Schweizerdeutschen inspirierende Ansätze vor. In einem wird das wh-doubling als eine Angelegenheit von Clitic-doubling betrachtet (cf. Poletto/Pollock 2004). Im anderen wird von der w-Bewegung nach rechts ausgegangen (cf. Neidle et al. 2000).

\subsection{Clitic-doubling}

Einige Nordostitalienische Dialekte weisen ähnlich wie der Urner Dialekt wh-doublingKonstruktionen auf.
(13) a. $S^{\prime}$ a-lo fat che?
Illasi (Verona)
What has-he done what
'What has he done?'
b. Ndo e-lo ndat endoe?
Where is-he gone where
'Where has he gone?'

Die Verdoppelung ist nicht mit allen w-Elementen möglich: w-Phrasen vom Typ che $+N P$ und parché 'warum' sind ausgeschlossen. In Illasi gibt es Variation in der Wahl der w-Wörter: während die älteren Dialektsprecher nur [che, $\left.\mathrm{s}^{\prime}\right]^{15}$ akzeptieren, weiten es die jüngeren auf $n d o$ 'wo' und $c i$ 'wer' aus, und die Sätze mit [che, s']-Struktur werden von älteren Personen als rhetorisch oder als 'surprise'-Fragen interpretiert (cf. Poletto/Pollock 2004: 33). In Strukturen ohne Verdoppelung variieren die Dialekte danach, ob das satzinitiale oder das satzfinale wWort ('in situ') realisiert wird.

In ihrer Argumentation gehen Cecilia Poletto und Jean-Yves Pollock (2004: 4) von drei Annahmen bzw. Beobachtungen aus: Erstens, die Doubling- und 'in situ'-Fälle sind auf wElemente beschränkt, die eine kurze, phonetisch reduzierte und unbetonte, und eine lange,

\footnotetext{
15 s' ist die Kurzform von cossa 'was'.
} 
phonetisch substanzreichere Form, haben. Es wird angenommen, dass es so etwas wie wKlitika gibt. Zweitens, wh-doubling und wh-in situ sind obligatorisch von SCLI (subject clitic inversion) begleitet. Drittens, in allen Doubling-Fällen steht das 'kurze' w-Wort links, das 'lange' rechts vom Verbkomplex.. Ferner wird das wh-doubling als eine Art Clitic-Doubling angesehen, wie z. B. im Französischen Il me parle à moi. So werden das klitische w-Wort und das nicht reduzierte w-Wort als eine komplexe Einheit [ClP whP wh-cl] in die Derivation eingefügt. Nach einigen Verkettungsoperationen ${ }^{16}$ gelangt die Derivation der Struktur mit SCLI zur linken Peripherie, die in diesem Fall mindestens aus drei Projektionen bestehen soll:

(14) $\left[\mathrm{WhP} 1 \mathrm{Wh}^{\circ} 1\left[\mathrm{ForceP}^{\circ}\left[\mathrm{WhP} 2 \mathrm{Wh}^{\circ} 2[\mathrm{IP} \ldots]\right]\right]\right.$

So landet am Ende der Derivation das w-Klitikon in WhP1, das andere w-Wort in WhP2 und die IP via remnant movement in ForceP, $d$. h. beide w-Elemente befinden sich im CP-Bereich. Auf diese Weise wird dafür argumentiert, dass in 'scheinbar' in situ-Fällen das w-Wort gar nicht in situ steht, sondern sich in der linken Peripherie befindet. Die Variation in romanischen Sprachen (wh-doubling, wh in situ und wh initial) ist damit ein und dasselbe Phänomen, bei dem die CIP unterschiedlich realisiert wird: ${ }^{17}$
a. S'a-lo fat che?
[ClP whP wh-cl] Illasi (Verona)
'What has he done what'
What has he done?

b. et fà què?

[ClP whP Ø] Monno

'Has-he done what'

What has he done?

c. $Q u^{\prime}$ a-t-il fait?

[ClP Ø wh-cl] French

'What has-t-he done'

What has he done?

Was das Schweizerdeutsche betrifft, gehe ich von der kopffinalen VP und IP und kopfinitialen Struktur der CP aus. In der minimalistischen Syntax kommen die w-Fragen in nicht in situ Sprachen durch die w-Bewegung zustande. Das w-Element wird in die Argumentposition in der VP eingefügt. Wenn die CP projiziert ist, wird es in die [Spec,CP]Position bewegt. In der CP werden die [wh]-Merkmale der bewegten DP und des C-Kopfes gecheckt. Bei den Sätzen mit wh-doubling stellt sich die Frage, welches die Position des wWortes am rechten Satzrand ist. In der Clitic-doubling Analyse wird das Problem gelöst, indem man für die zwei w-Wörter, die zusammen eine Clitic-Phrase bilden, zwei verschiedene Positionen (WhP1 und WhP2) in der CP annimmt.

Interessant an dieser Analyse ist die Annahme der klitischen w-Pronomina. Während in Illasi (Verona) der Unterschied zwischen klitischen und nicht-klitischen Pronomina gut sichtbar bzw. hörbar ist ( $s$ 'vs. che 'was', ndo vs. endoe 'wo'), ist das für das Schweizerdeutsche etwas

\footnotetext{
${ }^{16}$ Für den genauen Vorgang s. Poletto/Pollock (2004: 24-25).

${ }^{17}$ Der Analyse von Poletto/Pollock (2004) lehnt sich an Kayne (2005) an, wo die Unterschiede zwischen eng verwandten Sprachen beispielsweise dadurch erklärt werden, dass die einen den Kopf lexikalisieren, die anderen die Phrase.
} 
problematisch. Die einsilbigen w-Wörter am linken Rand werden normalerweise nicht betont, ausser in Echo-Fragen. Beispielsweise ist im Urner Dialekt der Unterschied zwischen zwei wKopien minim (cf. Abschnitt 2.1). Bei solchen w-Wörtern wie wiä 'wie', wo(o) 'wo' und weer 'wer', ${ }^{18}$ merkt man, wenn sie am linken Rand des Satzes stehen, dass der Vokal kürzer ausgesprochen wird, als wenn sie am rechten Rand stehen würden. Bei wenn 'wann', wass 'was' und wèmm 'wem' gibt es praktisch keinen Unterschied, ausser vielleicht, dass der Konsonant im Auslaut beim w-Wort am linken Rand geschwächt wird.

Klitische w-Pronomina würden im Übrigen in das pronominale Paradigma des UDs sehr gut passen: da weisen Personal-, Possessiv- und Demonstrativpronomina sowohl starktonige als auch schwachtonige Formen auf (cf. Clauss 1969: 102-106). Ansonsten gibt es keinen Grund, im Schweizerdeutschen klitische w-Pronomina anzunehmen. Mit der Frage der klitischen Pronomina hängt auch die Frage zusammen, wieviele w-Elemente in der syntaktischen Derivation beteiligt sind. Im Fall der ClP sind es zwei Elemente, die aus dem Lexikon in die Derivation eingeführt werden. Sie landen dann in zwei verschiedenen Positionen in der CP.

Bei der Annahme, dass im Lexikon ein w-Element zur Verfügung steht, kann das whdoubling durch die Bewegung und die Erhaltung der Kopie in situ erklärt werden, wie es in der Analyse für die ASL vorgeschlagen wird.

\subsection{W-Bewegung nach rechts: American Sign Language (ASL)}

Die ASL ist eine wh-in situ Sprache. Die w-Bewegung aber erfolgt nach rechts in die [Spec,CP]-Position, wie (16a) und (16b) zeigen: 19

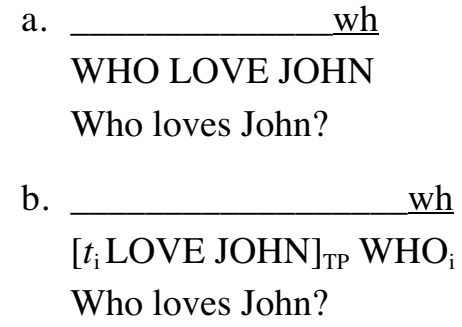

In der ASL sind Fragen mit mehr als einer w-Phrase, die demselben Argument entspricht, allgemein üblich (cf. Neidle et al. 2000: 114). Die Verdoppelung wird hauptsächlich in zwei recht unterschiedlichen Konstruktionen verwendet. In einem Fall befindet sich die eine wPhrase entweder in situ oder in [Spec,CP], und die andere wird in der tag-Position realisiert. Wie in anderen tag-Konstruktionen in ASL, kann in (17a) zwischen dem tag und dem Rest des Satzes eine kurze Pause eingelegt werden, darüber hinaus wird der tag von einer für die w-Wörter charakteristischen Kopfbewegung begleitet: ${ }^{20}$

\footnotetext{
${ }^{18}$ Die Schreibweise der w-Wörter ist der Grammatik des Urner Dialekts von Walter Clauss (1969) entnommen.

${ }^{19}$ Die Zeile mit den Kapitälchen gibt die Zeichen mit den Händen wieder (main gloss). Die Notation in Kleinbuchstaben über dieser Zeile zeigt die nicht-manuellen Bewegungen (z. B. mit dem Kopf oder mit den Augen). Die Linie signalisiert die Ausdehnung dieser Bewegungen in Bezug auf das main gloss. wh steht für die w-Frage.

${ }^{20} \mathrm{hs}$ in (17a) bedeutet head shake.
} 
(17)

a. hs

wh

WHO LIKE JOHN, WHO

Who likes John, who (does)?

b. wh

"WHAT," JOHN BUY "WHAT"

What, what did John buy?

In einem anderen Fall, s. (17b) steht das eine w-Wort in der Topik-Position als base generated topic am linken Rand. Es ist das Zeichen für 'was' und es kann mit allen anderen wPhrasen verwendet werden. Das andere w-Wort kann in situ bleiben oder nach rechts in die CP bewegt werden, wobei die letzte Option bevorzugt wird. Zwischen dem wh-topic und der w-Frage gibt es keine Pause (cf. Neidle et al. 2000: 115-117).

In der ASL ist die Situation insofern anders als in norditalienischen Dialekten (s. 5.1), als in (17a) nur ein w-Wort im Lexikon zur Verfügung steht. Nach der Bewegung der w-Phrase in die CP wird die Kopie in situ nicht gelöscht. Das ist für die tags üblich, wenn man annimmt, das sie eine reduzierte Wiederholung des Matrix-Satzes darstellen. So erfolgt das eigentliche wh-doubling in der Syntax (Kopierprozess) und nicht im Lexikon (CIP). Die Analyse der wPhrase am rechten Rand als tag würde einerseits gut zu waseliwas-Sätzen im Schweizerdeutschen passen, da diese das typische prosodische Muster für tags zeigen, nämlich eine Pause bzw. die Möglichkeit einer Pause vor der tag-Sequenz. Andererseits aber ist waseliwas nicht ganz eine Kopie des w-Wortes am linken Satzrand was. So kommt waseliwas wohl nicht durch eine Bewegung an den rechten Rand, sondern z. B. durch eine Adjunktion von rechts.

Interessant und ungewöhnlich an der Analyse von ASL ist die Idee der w-Bewegung nach rechts. Nach Auffassung von Carol Neidle et al. (2000) ist die [Spec,CP]-Position in der ASL rechts von C, d. h. die w-Bewegung erfolgt nach rechts. Diese Option ist zwar theoretisch möglich, praktisch wird sie aber von ganz wenigen Sprachen benutzt. Unter Sprachen, die diese Option benutzen, sind beispielsweise einige Dialekte des Tscherkessischen und des Abchasischen (beides kaukasische Sprachen mit SOV). ${ }^{21}$ In (18a) steht das w-Wort in situ. Solche Fragesätze stellen eine gefühlsbetonte Frageform dar (vgl. (6) \& (7)). ${ }^{22}$

Emphatisch unmarkiert ist dagegen (18b), eine Cleft-Konstruktion, in der die w-Phrase an den rechten Rand bewegt wurde (cf. Colarusso 1979: 41):23
a. (!)え'ə-m śəd (уə-)ə-šk-ə-ya-ra-r
Tscherkessisch

man-obl what (what-)he-eat-trans-past-part-abs

'What did the man eat (that time)?'

\footnotetext{
${ }^{21}$ Ich danke Josef Bayer für diesen Hinweis und für die Diskussion zu diesem Thema.

22 ! bezeichnet eine Emphase.

${ }^{23} \mathrm{Im}$ Tscherkessischen drückt die Cleft-Konstruktion in Deklarativa die Emphase oder den Fokus aus, nicht aber in w-Fragesätzen (cf. Colarusso 1979: 41).
} 
b. え'ə-m (уə-)ə-šk-ə-ya-ra-r śəd- $\varnothing$ man-obl (what-)he-eat-trans-past-part-abs what-abs 'What is it that the man ate (that time)?'

Die Cleft-Sequenz in (18b) besteht aus dem w-Wort und einer Kopula, die in w-Fragen nicht realisiert werden muss (im Beispiel nicht notiert). An diese Kopula wird laut John Colarusso (1979: 65) das w-Wort klitisiert.

Auch in dieser Analyse wird, wenn nicht explizit klitische w-Pronomina, so doch deren Klitisierung angenommen. Es ist in der Tat nicht ganz klar, wie die einsilbigen w-Wörter zu kategorisieren sind. Sind es Köpfe oder Phrasen? Kann ein w-Wort an einen ganzen Satz klitisiert werden?

Bei den Sätzen mit w-Verdoppelung gibt es grundsätzlich zwei Probleme. Erstens, wie und wo entstehen die Kopien der w-Wörter? Diese können als zwei separate Elemente in Form von einer Clitic Phrase (cf. Poletto/Pollock 2004) in die Syntax eingeführt werden, oder es wird eine Kopie durch Bewegung erzeugt, wobei die Spur in der Ausgangsposition nicht gelöscht wird (cf. Neidle et al. 2000). Zweitens, kann man von einer w-Bewegung nach rechts bzw. einer Adjunktion von rechts ausgehen?

\section{Zusammenfassung und Ausblick}

Die Existenz von wh-doubling im Schweizerdeutschen bekommt durch die Datenerhebung im Projekt 'Dialektsyntax' und durch die Texte aus dem Internet (waseliwas) eine weitere Bestätigung, da die bisherigen Beispiele lediglich aus dem Urner Dialekt stammen. Die wVerdoppelung findet in der ganzen Deutschschweiz mit Ausnahme des Berner Oberlandes eine niedrige Akzeptanz. Diese Frageform wird auch von den Sprechern anderer Dialekte in der Zentralschweiz als natürlichste Form bezeichnet. So gilt es als nächstes zu untersuchen, wie die w-Verdoppelung in diesen Dialekten realisiert wird. Dafür zeichnen sich nämlich zwei Muster ab. In einem Fall wird das w-Wort in Fokus-Funktion verdoppelt, wie im Urner Dialekt. Im anderen liegen rhetorische Kontexte vor, in denen die fragende Person ihren emotionalen Zustand oder den Informationsstand (von Vermutung bis Wissen) bezüglich der jeweiligen Gesprächssituation zum Ausdruck bringt.

Ferner zeigt sich, dass das Schweizerdeutsche nicht die einzige Sprache ist, in der das whdoubling und die w-Reduplikation (vgl. waseliwas) möglich ist. Die Untersuchungen zu diesen anderen Sprachen könnten unter Umständen neue Impulse für die Analyse des Schweizerdeutschen geben.

Für eine syntaktische Theorie stellen die Doubling-Phänomene immer noch ein Rätsel dar, besonders diejenigen, die die linke Peripherie betreffen. Beim wh-doubling stellt sich grundsätzlich die Frage, wie in der Syntax verdoppelt werden kann (z. B. Clitic-Phrase vs. Bewegung). Aufschluss darüber könnten auch die Analysen zu den extraponierten Elementen, wie z. B. die Extraposition von Komplementsätzen, geben. 


\section{Literaturangaben}

Boeckx, Cedric (2000): "Double Wh's". MIT Working Papers in Linguistics 38: 1-14.

Bucheli Berger, Claudia (2005): "Passiv im Schweizerdeutschen". Linguistik online 24, 3/2005: 49-77.

http://www.linguistik-online.de/24_05/bucheli.pdf.

Bucheli, Claudia/Glaser, Elvira (2002): "The Syntactic Atlas of Swiss German Dialects: empirical and methodological problems". In: Barbiers, Sjef/Cornips, Leonie/van der Kleij, Susanne (eds.): Syntactic Microvariation. Amsterdam: 42-74.

http://www.meertens. knaw.nl/books/synmic/.

Chung, Daeho (1999): "On WH-Doublets in Korean. Eoneohag Journal of the Linguistic Society of Korea 25: 281-301.

Clauss, Walter (1969): Die Urner Mundart. Ihre Laute und Flexionsformen. Altdorf.

Colarusso, John (1979): "Rightward movement, question formation and the nature of transformational process: the Circassian case". Papiere zur Linguistik 21/2: 27-73.

Erteschik-Shir, Nomi (1997): The Dynamics of Focus Structure. Cambridge.

Erteschik-Shir, Nomi/Strachov, Natalja (2004): "Focus structure architecture and P-syntax". Lingua 114: 301-323.

Fabricius, Anne H. (1998): A Comparative Survey of Reduplication in Australian Languages. München etc. (= Lincom Studies in Australian Languages 3).

Fischer, Ludwig (1960): Luzerndeutsche Grammatik. Zürich.

Frey, Natalja (2001): Wiederholung der w-Phrasen im Urner Dialekt des Schweizerdeutschen. Lizentiatsarbeit. Universität Bern.

Greyerz, Otto von (1976): Berndeutsches Wörterbuch. Bern

Haspelmath, Martin (1997): Indefinite Pronouns. Oxford.

Kayne, Richard (2005): "Some notes on comparative syntax, with special reference to English and French". In: Cinque,G./Kayne, Richard (eds.): The Oxford Handbook of Comparative Syntax. New York.

Munaro, Nicola (2003): Interrogative strategies in the Northen Italian domain. Handout. ESF/SCH Exploratory Workshop European Dialect Syntax, Padova, 11-13th September 2003.

Muster, Peter (2001): Baselbieter Wörterbuch. Basel

Neidle, Carol et al. (2000): The Syntax of American Sign Language. Cambridge.

Odermatt, Esther (1903): Die Deminution in der Nidwaldner Mundart. Zürich.

Penner, Zvi (1992): "The ban on parameter resetting, default mechanisms, and the acquisition of V2 in Bernese Swiss German". In: Meisel, Jürgen M. (ed.): The Acquisition of Verb Placement. Dordrecht: 245-281.

Poletto, C./Pollock, J.-Y. (2004): "On wh-clitics and wh-doubling in French and some North Eastern Italian dialects". Probus 16/2, 241-272.

Seiler, Adolf (1879): Die Basler Mundart: ein grammatisch-lexikalischer Beitrag zum schweizerdeutschen Idiotikon: zugleich ein Wörterbuch für Schule und Haus. Basel.

Suter, Rudolf (1976): Baseldeutsch-Grammatik. Basel.

Suter, Rudolf (1995): Baseldeutsch-Wörterbuch. Basel. 
Strawson, Peter Frederick (1964): "Identifying reference and truth-values". Theoria 30: 96-118.

Weber, Albert (1948): Zürichdeutsche Grammatik. Zürich.

Yamakoshi, Kyoko (2002): "Wh-drop in child languages and adult ASL". In: van Koppen, Marjo et al. (eds.): Proceedings of Console IX. Leiden: 217-231. 